\title{
El modelo de la enseñanza-aprendizaje del Derecho Penal
}

\author{
The Criminal Law teaching-learning

\author{
Samuel Rodríguez Ferrández \\ Profesor Ayudante Doctor de Derecho Penal y de \\ Criminología. Departamento de Historia Jurídica y \\ Ciencias Penales y Criminológicas. \\ Universidad de Murcia. \\ Email: samuel.rodriguez@um.es
}

Elena B. Fernández Castejón

Profesora Asociada de Derecho Penal.

Departamento de Ciencia Jurídica.

Universidad Miguel Hernández de Elche.

Email: efernandez@umh.es

\section{Resumen}

El objetivo del presente trabajo es el de transmitir cuál es nuestro propio modelo de enseñanza-aprendizaje del Derecho Penal a través de la exposición de los propios fundamentos que lo inspiran para, a continuación, explicar las técnicas de metodología docente teórica y práctica que implementamos (incluyendo los sistemas de evaluación) y los recursos empleados, haciendo referencia puntual a su vez a diferentes experiencias innovadoras en las que hemos participado. Ello nos llevará a concluir, de un modo reflexivo, sobre la necesidad de ser conscientes, como docentes de enseñanza superior, sobre la constante innovación que requiere nuestra labor para adaptarse continuamente a nuestra cambiante realidad. 


\section{Palabras clave}

Derecho Penal; enseñanza; aprendizaje; metodología docente; espacio europeo de educación superior.

\section{Abstract}

This paper pretends to convey which is our own Criminal Law teaching-learning model through the statement of reasons that inspire and, then, explain the techniques of theoretical and practical teaching methodology that we implement (including evaluation systems) and the resources we use, explaining along different innovative experiences in which we have participated. Then, we will be able to conclude, in a thoughtful way, about the need to be conscious, as teachers of higher education, about the constant innovation which requires our efforts to be continually adapted to our changing reality.

\section{Key words}

Criminal law; teaching; learning; teaching methodology; european higher education area. 


\section{Consideraciones generales sobre la enseñanza del Derecho penal}

\subsection{Estado actual de la enseñanza universitaria en Derecho penal}

De nada sirve definir el qué debe impartirse en las asignaturas de "Derecho penal. Parte general" y "Derecho penal. Parte especial" si no definimos el cómo debe realizarse esa labor. En efecto, la concreción de la propuesta docente no está finalizada con la definición del contenido, sino que resulta fundamental también concretar la forma en la que el mismo va a transmitirse a los estudiantes. Éste es, pues, el objetivo fundamental de este trabajo que, por tanto, para nada debe minusvalorarse, y menos en un momento como el actual, en el que, como veremos a continuación, en el que nos hemos visto necesitados de darle cada vez más importancia a las metodologías docentes.

Así, a pesar de que pueda parecer pretencioso para un apartado de un artículo como éste empezar con un retrato de cómo era hasta no hace mucho la enseñanza del Derecho penal en la Universidad española, creemos necesario comenzar este trabajo, precisamente, con una breve reflexión acerca de cuál era, en líneas generales, la situación de la docencia universitaria en Derecho penal hasta la implantación de los Grados. Sólo partiendo de la situación actual podremos, después, centrar el interés de este trabajo en tratar de dejar constancia de cómo, desde nuestro punto de vista, y tomando en consideración las reformas ya implementadas inspiradas en las directrices del nuevo "Espacio Europeo de Educación Superior", debe ser la docencia en Derecho penal actual y del futuro. Para ello trataremos de concretar cuáles son las líneas básicas que deben servir de orientación para la tarea docente, en aras a que ésta tenga la mayor calidad que sea posible, y a que, en consecuencia, los futuros actuales y futuros egresados en Derecho logren un mejor conocimiento sobre la Ciencia del Derecho en general, y del Derecho penal en particular ${ }^{1}$.

Pues bien, no creemos que sea una afirmación excesivamente arriesgada la de que la situación de la enseñanza del Derecho dentro de las universidades españolas estaba lejos de ser la ideal. Estamos totalmente de acuerdo con las principales conclusiones en ese sentido adoptadas en su momento por el Profesor GONZÁLEZ RUS que, en su acertado análisis del "hoy de la enseñanza del Derecho", realizaba una dura y crítica revisión de la Universidad española en general, y de la enseñanza del Derecho penal en particular, que le llevaba a remarcar la necesidad de cambio, tanto en las formas y contenido de la docencia, como en muchas de las estructuras e instituciones de la propia Universidad. Así, de entre las diferentes variables que exponía que representaban un anticuado método de enseñanza, se encontraban elementos tales como los sigui-

\footnotetext{
${ }^{1}$ A este respecto es interesante destacar el trabajo, de ya hace más de una década, de GONZÁLEZ RUS (2003). En este artículo, el autor describía tanto el panorama entonces vigente en la enseñanza del Derecho penal, como la forma en que debía realizarse dicha enseñanza. También, incluso antes, los Profesores BOIX REIG y ORTS BERENGUER (1997: 210-215) realizaron un análisis de los problemas de la situación de nuestras facultades, quizá con mayor pesimismo que el trabajo antes comentado, en relación con aspectos tan diversos como la organización de muchos departamentos, la estructura y contenido de los planes de estudio, o la situación de desánimo del profesorado, además de su carencia en preparación pedagógica.
} 
entes: el papel central, si no único y exclusivo, que para el profesor universitario cumplía la docencia teórica, basada ésta en la lección magistral, con lo que ello implica de anulación total de cualquier participación activa por parte del estudiante; el sistema de evaluación de las materias, por lo menos en las asignaturas de Derecho, dado que la misma se realizaba teniendo en cuenta básicamente la capacidad memorística de los alumnos, obviando así una valoración de su capacidad crítica y creativa; relacionado con ello, también criticaba con razón el autor que fueran los exámenes el eje central sobre el que construir el sistema educativo, dado que ello favorecía claramente el absentismo de los alumnos e impedía que obtuvieran otros beneficios, distintos a los del propio título oficial, posibles dentro de la vida universitaria; por último, y con respecto a los programas y contenidos docentes, consideramos muy acertada la crítica de GONZÁLEZ RUS, en la medida que advertía de la sobrecarga de los mismos, encontrándose los planes de estudio de las entonces Licenciaturas más orientados a la acumulación de aspectos conceptuales, y en muy poca o escasa medida a la solución de problemas, convirtiéndose así el modelo universitario actual español en un modelo que primaba la cantidad de temas tratados antes que la intensidad en el análisis y estudio de los mismos (GONZÁLEZ RUS, 2003: 3-9). No hay duda alguna de que éste es un análisis parcial del problema entonces detectado, y que no se comparaba con la situación precedente, en la que estos defectos aún estaban más acentuados. Pero es poco consuelo el yerro pasado, y entendemos que los datos anteriormente señalados servían de base desde la que repensar cómo debía construirse una mejor plataforma para la docencia en Derecho penal.

$Y$ es que, y aunque resultaba poco alentador este panorama de la docencia universitaria que, además, era probablemente aplicable a la mayoría de las titulaciones de la Universidad española, es cierto que desde hace unos años empezamos a tomar conciencia del problema y, por tanto, de lo importante que puede ser para el futuro de una sociedad la consecución de un buen sistema de docencia universitaria. Por ello, y como señalaba, de nuevo con acierto, GONZÁLEZ RUS, por donde había que empezar era por una concienciación del profesorado sobre esta necesidad de cambio y realizáramos todos los esfuerzos que estuvieran o estén en nuestra mano para que la docencia universitaria tenga el prestigio que merece. En esta línea, pues, y tras describir someramente las directrices generales que empezaron a guiar los estudios de Derecho hace unos años, marcadas por el "Espacio Europeo en Educación Superior", centraremos este trabajo en proponer una metodología docente aplicada al ámbito del Derecho penal, tomando en consideración las propuestas que los psicólogos y pedagogos (como expertos en el estudio de la enseñanza-aprendizaje) nos hacen, para mejorar la docencia universitaria en Derecho penal.

\subsection{Objetivos generales y específicos de la enseñanza del Derecho penal}

Decía el Profesor TORíO LÓPEZ (2004: 917), en uno de los pocos trabajos que hasta hacía unos años había dedicado algún componente de la doctrina del Derecho penal a la enseñanza del Derecho, que "la Facultad universitaria de Derecho tiene como misión la investigación de la creación del Derecho y, sólo ulteriormente, de la aplicación del Derecho", así como que, en este sentido, resultaba (y todavía lo es) bastante desalentador observar cómo algunas de las más importantes reformas penales se llevan a ca- 
bo al margen de la Ciencia del Derecho o de la doctrina universitaria del Derecho. Con ello quería reflejar el autor que parte de la responsabilidad por la pérdida de importancia social de la titulación de Derecho nos correspondía, precisamente, a los docentes, que nos empeñábamos en transitar los caminos equivocados. De hecho, todos coincidiremos en que nuestros estudiantes de Derecho deben tener una sólida formación teórica del Derecho, siendo la misión de los profesores el estimular y desarrollar las competencias, habilidades o técnicas que caracterizan propiamente el trabajo del jurista (PÉREZ LLEDÓ, 1992: 81-82).

Por ello, siguiendo la filosofía con la que pretendemos que quede reflejada nuestra idea de la docencia a lo largo del presente trabajo, y asumiendo por un lado la idea de lo absurdo que resulta la utilización de procedimientos memorísticos acerca de normas $y$, también, de doctrinas jurídicas, y, por otro, la necesidad de la formación e incorporación de la utilización de las nuevas tecnologías en nuestra disciplina, consideramos que puede definirse como objetivo central de la enseñanza del Derecho aquél de "ejercitar a los alumnos en las técnicas que les permitan conocer el Derecho, instruyéndolos en los métodos y procedimientos de autoaprendizaje que los capaciten para realizar por sí mismos la interpretación y aplicación de los principios y normas jurídicas, tanto las vigentes como las que se producirán en el futuro" (GONZÁLEZ RUS, 2003: 10).

Es decir, el objetivo general de las Facultades de Derecho debe ser el de enseñar a pensar como juristas, y no como "abogados", "jueces" o "candidatos a funcionarios". Por mucho que a veces nos pueda parecer que la actual cultura de la "calidad universitaria", en ocasiones mal entendida por quienes la fomentan, nos Il eva a tener más en cuenta la inserción laboral inmediata que la formación integral del jurista, una formación que debería estar inspirada en valores esenciales para la convivencia (ABEL SOUTO, 2010). De este modo, debe quedar fuera de toda duda que nuestras Facultades, y en contra de lo que parece que ocurre en las academias que preparan oposiciones, o en los bufetes que adquieren "pasantes" para determinadas tareas, no deben perseguir que sus egresados sean repetidores autómatas de textos legales. Nuestros egresados puede que tengan que aprender todo ello después, pero antes deben saber leer y analizar críticamente la Ley, la de ahora, la del futuro... la que sea; lo cual, sin duda, es mucho más difícil y sólo puede enseñarse en las universidades.

Este objetivo general nos debe llevar, también, a los objetivos específicos, que en el lenguaje actual de la calidad se denominan "Competencias" a fomentar a lo largo de la formación de los futuros egresados en Derecho. En este sentido, parece claro que deben ser objetivos docentes aquellos de lograr que los estudiantes conozcan los principios y normas reguladoras del Derecho penal, para así, siendo capaces de conocer y utilizar las diferentes herramientas y técnicas que los capaciten en la búsqueda y solución de planteamientos teórico-prácticos, poder realizar por sí mismos la interpretación y aplicación de los principios y normas penales, tanto de las vigentes, como de las que se producirán en el futuro. Además, deberán aprender a utilizar las herramientas informáticas y tecnológicas que les faciliten su tarea; deberán empezar a fortalecer su capacidad crítica oyendo opiniones diferentes y viéndose obligados a defender las suyas; deberán ser capaces de presentar trabajos, realizar exposiciones orales y reali- 
zar trabajos en equipo; y todo ello, siempre, desde el respeto a sus profesores y compañeros y compañeras.

\section{Métodos y procedimientos para la enseñanza universitaria de Derecho penal}

\subsection{Nuevas perspectivas de enseñanza universitaria en Derecho penal y aprendizaje}

El proceso de Convergencia Europea no sólo se ha dirigido hacia la armonización de las titulaciones, sus cursos y sus contenidos (MORILLAS CUEVA, 2013: 11-44), sino, también, hacia la mejora de los procesos y metodologías docentes. Quizás, y fuera de otro tipo de consideraciones políticas, lo mejor que haya podido ofrecer el proceso de BoIonia sea esto: una toma de conciencia de la importancia de la mejora docente $y$, concretamente, un cambio en el concepto de docencia; de la enseñanza a la enseñanzaaprendizaje. Tomando en consideración las propuestas acerca de la metodología docente a aplicar que se realizaban ya en el "Libro Blanco del Título de Grado en Derecho" ${ }^{2}$, y tal y como indican los expertos en el proceso de aprendizaje, el estudiante debe tener un papel protagonista, adoptando un rol mucho más activo en este proceso. Veamos ahora, pues, cuáles son los distintos instrumentos metodológicos de los que disponemos para lograr estos propósitos.

Dentro de las teorías de la Psicología del aprendizaje, aquéllas que destacan este papel más activo del sujeto en la relación enseñanza-aprendizaje son las que se basan en los supuestos de la psicología cognitiva ${ }^{3}$. De entre las principales teorías que nos ofrece la Psicología, vamos a destacar dos de ellas: el aprendizaje por descubrimiento, y el aprendizaje significativo. La razón para centrarnos en estas dos teorías y no en otras es doble. Por una parte, se trata de dos teorías que se han orientado a la aplicación en la enseñanza. Por otra parte, y además de ajustarse en gran medida a la idea planteada a lo largo de este trabajo, acerca de la necesidad de utilizar distintosinstrumentos y metodologías para la mejora docente, ofrecen una visión distinta de cómo enfocar la docencia, pero, al mismo tiempo, son complementarias en su valoración del papel activo que ha de tener el estudiante en el proceso de enseñanza-aprendizaje. Por supuesto ni

\footnotetext{
${ }^{2}$ Con la introducción del sistema de créditos "ECTS", se pretendía, entre otras, una sustancial disminución de las clases presenciales en beneficio de los seminarios, las tutorías y el trabajo individual a cargo de los alumnos. Tal y como afirman los expertos, para conseguir todos los objetivos propuestos, se advertía la necesidad de emprender una serie de medidas que garantizasen la viabilidad del modelo propuesto, entre las que destacan: la revisión, actualización y adecuación a dicho modelo de los contenidos de cada una de las asignaturas integradas en el plan de estudios; el reajuste del "ratio" entre estudiantes y profesor mediante una notable reducción del tamaño de los grupos de clase, tanto en las clases teóricas como en las prácticas; el aumento en el gasto corriente previsto por alumno matriculado; la ampliación del colectivo de docentes doctores y numerarios; el fomento de las iniciativas dirigidas a la introducción de innovaciones y mejoras pedagógicas o el creciente apoyo a la movilidad interuniversitaria de los estudiantes a través de programas como el "Erasmus", o el actual "SICUE"; así, como, por supuesto, la necesidad de que estas medidas vayan acompañadas de la financiación necesaria para sostenerlas ("Libro Blanco del Título de Grado en Derecho", Parte segunda, p. 149). Precisamente, se ha escrito sobre la importancia formativa del programa "Erasmus" en el ámbito de las Ciencias Penales y Criminológicas, tanto desde el punto de vista de la movilidad estudiantil (CARIO/DE LA CUESTA ARZAMENDI, 1990), como desde el relativo a la movilidad docente (BERNAL DEL CASTILLO, 2014).

${ }^{3}$ Los supuestos del aprendizaje cognitivo se basan en que los procesos mentales van cambiando con el aprendizaje, rompiendo con las tradiciones más conductistas que enfatizaban el aprendizaje como un mero cambio de conducta. Así para los psicólogos cognitivistas, el conocimiento no es una simple acumulación de datos. La esencia de la adquisición del conocimiento estriba en el aprendizaje de relaciones generales (PUENTE FERRERAS, 2003: 259).
} 
pretendemos ni podemos desarrollar, como si fuéramos pedagogos, teorías sobre el aprendizaje, por lo que no debe interp retarse este apartado en este sentido, pero sí hemos querido, desde los escasos conocimientos que hemos obtenido en distintos cursos de formación docente, profundizar algo más en las distintas posibilidades existentes para que la enseñanza sea más productiva para el estudiante. Lo que hemos encontrado en este sentido es que utilizamos muchas más técnicas de lo que creemos, pero, en todo caso, ello no nos debe llevar a pensar que sabemos hacerlo todo y que no podemos mejorar.

\section{A) Teoría del aprendizaje por descubrimiento}

Jerome S. BRUNER (1961) es uno de los psicólogos que más ha influido en el campo evolutivo y en la teoría del desarrollo. BRUNER estaba interesado en las relaciones entre desarrollo, la enseñanza y el aprendizaje, y plantea que los profesores deben proporcionar situaciones problemáticas que estimulen a los alumnos a descubrir las estructuras de las asignaturas. Para BRUNER, el aprendizaje es un proceso constante de obtención de una estructura cognitiva que representa el mundo físico e interactúa con él (PUENTE FERRERAS, 2003: 269-270). Según BRUNER, aprender por descubrimiento quiere decir obtener uno mismo los conocimientos: probar y formular hipótesis antes que simplemente leer o escuchar las lecciones. Plantea que descubrir es una forma de razonamiento inductivo porque los estudiantes formulan reglas, conceptos y principios generales, $y$, por ello, entre los beneficios del descubrimiento está el fomento del aprendizaje significativo. BRUNER cree que el aprendizaje debería tener lugar inductivamente, desplazándose desde los ejemplos específicos presentados por el profesor a generalizaciones acerca de la materia en cuestión que son descubiertas por los estudiantes. Creemos que estas ideas, del influyente psicólogo americano en el mundo de la educación, pueden servir para la docencia en Derecho penal. Veamos un ejemplo: partiendo de ejemplos diferentes de cada una de las causas de justificación, aparentemente de distinta naturaleza entre sí y con diferente fundamento, puede llegar a entenderse de forma más clara el fundamento genérico de las causas de justificación como conflicto de intereses; ello a su vez, podría permitir después, a los estudiantes, una mejor comprensión de la diferencia entre la proporcionalidad en el resultado del estado de necesidad frente a la proporcionalidad en los medios de la legítima defensa. Todos los profesores de Derecho penal que nos preocupamos por la docencia hemos utilizado este tipo de instrumentos del aprendizaje por descubrimiento en más de una ocasión. Pero consideramos que deben planificarse las clases teniendo en cuenta no sólo qué contenido va a impartirse sino, también, cómo, y las técnicas de BRUNER pueden servir en más de un caso para que el estudiante aprenda más y mejor.

\section{B) Teoría del aprendizaje significativo}

Para David Paul AUSUBEL (1963) el aprendizaje significativo consiste en la adquisición de ideas, conceptos y principios al relacionar la nueva información con los conocimientos en la memoria. El aprendizaje es significativo cuando guarda una relación sistemática con los conceptos pertinentes de la memoria a largo plazo; así el nuevo material amplía, modifica o elabora la información de la memoria. AUSUBEL, al contrario que BRUNER, propugna una estrategia deductiva para la enseñanza, teniendo que pre- 
sentarse por parte del profesor el material muy bien estructurado, organizado y de forma secuencial, proponiendo así su modelo de enseñanza por exposición, donde además de un estrecho contacto entre profesor y estudiante, se requiere la utilización de muchos ejemplos y que los conceptos generales se presenten primero y los específicos sean derivados de ellos. Para AUSUBEL, el docente debe, sobre todo, enseñar a pensar (PUENTE FERRERAS, 2003: 271). Esta estrategia de aprendizaje se puede aplicar perfectamente en muchos aspectos de nuestras asignaturas de Derecho penal; así por ejemplo, para la explicación de la diferente naturaleza de los dos juicios esenciales del delito (antijuridicidad y culpabilidad), y antes de la explicación específica de cada uno de sus elementos, puede resultar valioso remarcar la diferente naturaleza de la función de la norma en uno y otro caso, así como que ello vaya siendo completado con ejemplos derivados de las distintas causas de exclusión de la pena del art. 20 CP 1995 (cuya naturaleza en unos casos es de ausencia de antijuridicidad y en otros es de ausencia de culpabilidad).

\subsection{Metodología docente para la enseñanza universitaria en Derecho penal}

Tal y como afirma el catedrático del área de Didáctica de las Ciencias Experimentales, Fermín Mạ GONZÁLEZ GARCÍA, toda propuesta docente ha de inscribirse en un marco pedagógico que suponga una reflexión y revisión acerca de cuál es la mejor forma de aprender y enseñar ${ }^{4}$. Por tanto, y atendiendo a la relación que entre ambos conceptos proponen diferentes autores, enseñar significa planificar un programa educativo atendiendo a aquellos elementos que facilitan el aprendizaje: objetivos, metodología y evaluación.

En función de ello, el profesor puede adoptar una metodología u otra, d ependiendo de la elección que haga, pudiendo oscilar entre las centradas en el profesor o en el estudiante ${ }^{5}$. Como se podrá imaginar, la adopción de una u otra forma de enseñanza o las combinaciones de éstas dependerán de su adecuación a los objetivos, características de los estudiantes, exigencias de la asignatura y contexto, entre otras. Teniendo ello en cuenta, estamos de acuerdo con la Profesora DE LA CRUZ TOMÉ (1981) en que se ha de "encontrar el punto de equilibrio entre la enseñanza basada en el profesor o en el estudiante, $y$, por otro, combinar la enseñanza en la transmisión de información con la resolución de problemas en el aula, utilizada esta última en los casos prácticos en los que se aplican los conocimientos teóricos transmitidos".

Partiendo de estos principios, vamos a presentar una breve exposición de los elementos que tradicionalmente son consideradosimportantes por los expertos para impartir la docencia, para, después, hacer una aplicación de los mismos a la docencia específica de las asignaturas de Derecho penal.

${ }^{4}$ GONZÁLEZ GARCÍA, IBÁÑEZ y CASALÍ (2000:255) definen el aprendizaje como "los cambios relativamente estables en la conducta y conocimientos del alumno como resultado del programa de instrucción; mientras que la enseñanza es el conjunto de decisiones, actividades y medios organizados para facilitar el aprendizaje y promoverlo".

${ }^{5}$ Unas formas enfatizan la enseñanza basada en la resolución de problemas donde el alumno parte de una situación concreta, a partir de la cual va adquiriendo los conocimientos básicos para solucionarlo y entenderlo o bien basada en la transmisión de información, donde el profesor planifica los contenidos que son transmitidos oralmente y, sobre esta base, el alumno organiza y utiliza dichos conocimientos. 
A la hora de elaborar un programa docente, no ya en el sentido del contenido, sino en el de la programación docente, el punto de partida debe ser, sin duda, la concreción de los objetivos docentes. El proceso por el que se inicia nuestro trabajo es por la formulación de objetivos, es decir, por la definición de aquello que queremos que aprendan nuestros alumnos. Los objetivos pueden ser generales o específicos; los primeros constituyen un marco de referencia inicial y se orientan a la planificación, pero no equivalen a una conducta directamente observable. Los objetivos específicos u operativos se formulan en términos de lo que se puede evaluar, y permiten contrastar los aprendizajes que se desean alcanzar en el alumno. Así, en las guías docentes de las asignaturas los objetivos han sido formulados siguiendo estas directrices. Y el camino a seguir para conseguir dichos objetivos o fines propuestos, es la denominada metodología docente, que implica una serie de principios, reglas y técnicas para ponerlos en práctica de una manera racional y ordenada. A continuación describimos los métodos o técnicas de enseñanza más habituales tanto para la enseñanza teórica como para la práctica (DE PABLOS PONS, 1988), y que, según sea el caso, indicaremos su aplicabilidad en las asignaturas propias del área de Derecho penal.

\subsubsection{Metodología en la enseñanza teòrica}

El principal método utilizado para la enseñanza teórica es la exposición, que consiste en la transmisión de información por parte del profesor. Habitualmente, incluye las técnicas de la lección magistral, la lección magistral participativa, los mapas conceptuales y las sesiones de demostración. Tanto la lección magistral como la lección magistral participativa comparten la característica común de la transmisión oral que realiza el profesor de sus conocimientos, permitiéndole enfatizar los puntos más importantes de la materia, estimular el interés y la motivación del alumno. Además, permiten enseñar a un gran número de estudiantes a la vez. La lección magistral participativa pretende suplir la deficiencia del poco protagonismo del estudiante en la lección magistral tradicional con la estimulación del diálogo y la utilización de preguntas que llamen la atención del mismo, y por tanto, el profesor ha de formularlas pensando en su relevancia para el estudiante. Ésta es la técnica que consideramos personalmente más útil para realizar una gran parte de la docencia teórica en Derecho penal, principalmente en los dos extremos temporales: en el principio de la docencia al iniciar el curso académico para que los estudiantes empiecen a percibir las diferencias existentes entre lo que un lego en materia jurídica opine de las distintas instituciones penales, y lo que ellos, como futuros juristas, deben saber de esos mismos conceptos. También en las últimas clases del curso académico creo que es esencial la participación del alumnado, dado que es cuando ellos mismos deben empezar a percibir hasta qué punto entienden el sistema del delito. En definitiva, es necesario que los estudiantes sigan las directrices marcadas por el profesor, y que, al mismo tiempo, sea éste el que vaya motivando a los alumnos con preguntas, y utilizando éstas para que vayan aprendiendo a relacionar conceptos e ideas.

Otra de las técnicas para la exposición de contenidos es la de los mapas conceptuales, que, como afirman GONZÁLEZ GARCÍA, IBÁÑEZ y CASALÍ, la podemos utilizar como 
técnica innovadora para la mejora del proceso de enseñanza-aprendizaje ${ }^{6}$. El mapa conceptual se utiliza como un instrumento válido para representar la estructura conceptual de una disciplina o parte de la misma, pues facilita mediante la expresión gráfica las relaciones proposicionales entre conceptos. Algunas de las reglas generales para su uso y confección son las siguientes ${ }^{7}$ :

- Identificación de los conceptos clave del tema a exponer sugiriendo un límite de doce.

- Orden conceptual según un criterio de mayor a menor generalidad.

- Finalizar con los conceptos más específicos y apoyarse con el uso de ejemplos.

- Unir los conceptos gráficamente y utilizar en su caso palabras de enlace que definan sus relaciones.

Quizás no en sentido estricto, pero sí en cuanto a sus directrices generales, puede encuadrarse en el concepto de mapas conceptuales la técnica que nosotros utilizamos para, durante las cuatro primeras clases, explicar el esquema general del delito y cada uno de sus elementos, y que nos lleva, el último de esos días, a realizar conjuntamente el esquema del delito que después deberán imprimir y utilizar los estudiantes en el resto de las clases.

Los trabajos individuales o en grupo, constituyen otro instrumento de la docencia teórica que ofrecen un contacto directo con el material a aprender, satisfaciendo en parte el objetivo de individualización de la enseñanza. Para lograr una eficacia adecuada deben de realizarse bajo la dirección del profesor, $y$, normalmente en las horas de tutoría. A pesar de estar de acuerdo con la idea de que esta técnica puede ser muy útil para la individualización de la enseñanza, en nuestro caso la utilizamos más como una parte de la evaluación de las prácticas de las asignaturas que como un método docente en general.

La discusión, como última técnica docente a comentar para la docencia teórica, es un método que tiene como objetivo esencial el aprendizaje basado en la participación activa del alumno. Ha de estar previamente planificada y dirigida por personal competente; aunque suele estar indicada para grupos pequeños utilizando técnicas como seminarios y resolución de problemas, existen alternativas para grupos más numerosos, como, por ejemplo la técnica denominada foro. En ella se permite que grupos más numerosos discutan informalmente un tema dirigido por un coordinador. Suele realizarse a continuación de una conferencia, y se permite la participación de todos los presentes exponiendo sus ideas, opiniones y cuestiones. El coordinador debe controlar y organizar el tiempo para asegurar la eficacia de esta técnica, tanto el de quienes exponen el tema de la conferencia, como el de las personas del público que intervengan, así como de redirigir las preguntas que puedan desviarse del tema. En nuestro caso, en

\footnotetext{
${ }^{6}$ Se afirma que los mapas conceptuales son una buena herramienta empírica para la consecución de un aprendizaje significativo, que, tal y como se mencionó anteriormente, éste es entendido como la incorporación sustantiva de los nuevos conocimientos en la estructura cognitiva del sujeto (GONZÁLEZ GARCÍA, IBÁÑEZ y CASALÍ, 2000: 275).

${ }^{7}$ GONZÁLEZ GARCÍA, IBÁÑEZ y CASALÍ (2000: 276) proporcionan abundante información y explicaciones aplicadas para la confección de mapas conceptuales dirigidos tanto a la programación de asignaturas, o diseños de temas individuales.
} 
las clases teóricas, y dado que ante un grupo de estudiantes no muy numeroso (40-50) utilizamos esta técnica como paso previo, por ejemplo, para adoptar la teoría de la acción o para definir el fundamento y funciones de la culpabilidad en Derecho penal.

Como reflexión final de este punto de la docencia teórica queremos destacar, tal y como el Profesor GONZÁLEZ RUS afirmaba (2003: 14), que las clases se deben convertir "fundamentalmente en un tiempo y en un espacio dirigido, no a decir al alumno qué debe pensary saber, sino, sobre todo, a "enseñarle a pensar»". Así mismo, consideramos que en todas las técnicas que el profesor pueda utilizar para mejorar su docencia, es importante tener presente que entre nuestros objetivos están también que los estudiantes aprendan procedimientos y métodos de trabajo que los capaciten para identificar los problemas que se les planteen y saber resolverlos.

\subsubsection{Metodología en la enseñanza pràctica}

La enseñanza práctica se dirige a facilitar la adquisición de destrezas y procedimientos relevantes de la asignatura; aprender a utilizar el método científico en el área de conocimiento, entrenar en la resolución de problemas y/o desarrollar actitudes profesionales específicas ${ }^{8}$. Actualmente la enseñanza práctica está más representada en los nuevos planes de estudio ante un incremento en su valoración, y no sólo como parte inescindible de la docencia, junto a la de carácter teórico, sino también a través de la de la generalizada presencia de la asignatura "Practicum" en los títulos oficiales de Grado en Derecho (normalmente como materia obligatoria y sólo puntualmente como materia optativa).

Según los expertos en este ámbito, la enseñanza práctica dentro de una asignatura debe cumplir ciertos requisitos:

- Que la tarea práctica sea percibida por el estudiante como relevante y significativa para sus intereses.

- Que realmente facilite el aprendizaje cumpliendo los requisitos de retroalimentación y valoración de los resultados durante su proceso de realización.

- Que esté bien planificada y teóricamente bien fundamentada, siguiendo la coherencia de los contenidos teóricos de la asignatura.

Estos tres aspectos, a nuestro juicio, han de estar totalmente asumidos por parte del profesor a la hora de la realización de la guía docente de la asignatura, ya que, por desgracia, en muchas ocasiones las prácticas han sido valoradas únicamente como un "desahogo" por parte del profesor, que deja que el alumno pueda realizar un trabajo sin preparación teórica "pre" y "post" que debiera acompañar a la actividad. A continuación se describen brevemente algunas técnicas de la enseñanza práctica.

\footnotetext{
${ }^{8}$ DE LA CRUZ TOMÉ (1981) destaca en este sentido de la enseñanza práctica, que es la que constituye la faceta más difícil de llevar a cabo con éxito por el alto coste de material y tiempo que conlleva, es la que más se relaciona con los objetivos que se quieren conseguir, motiva al estudiante, y confiere sentido a la teoría.
} 
Ejercicios programados: consisten en trabajos estructurados diseñados para producir determinados resultados correctos, bien definidos, y encaminados a obtener una determinada respuesta. Con ellos se trata de demostrar o ilustrar los principios teóricos expuestos en clase o leídos por los estudiantes. En este punto está muy extendida en nuestro área de conocimiento (SÁINZ CANTERO, 1983-1984), y aceptada desde hace mucho tiempo, la utilización del denominado "método del caso", con la preparación previa por parte de los docentes de una guía orientativa sobre cómo proceder a la resolución del mismo (NIETO MARTíN, 2008), así como de unos materiales docentes específicos con ejemplos ya resueltos y formulación de otros casos para resolver (íNIIGO CORROZA/SÁNCHEZ-OSTIZ GUTIÉRREZ, 2014). En nuestro caso, hemos participado en la confección de este tipo de materiales, bien orientándolos a la docencia de forma planificada (PERIS RIERA et al., 2013), bien sea como resultado de otras técnicas, como el Seminario al que seguidamente haremos referencia (MIRÓ LLINARES/RODRÍGUEZ FERRÁNDEZ, 2010).

Seminarios: son sesiones de discusión sobre temas teóricos, dirigidos por el profesor, y constituyen una de las técnicas más difundidas para la enseñanza en grupos pequeños. Sus objetivos son: promover la comprensión; desarrollar la capacidad de razonamiento y crítica del estudiante; fomentar el que aprendan a pensar de una manera científica; y mejorar su habilidad en la solución de problemas. Lo propio del seminario es el enfoque en profundidad de un tema seleccionado por el grupo. Para que los seminarios sean efectivos deben estar estructurados y tener los objetivos claros. En una primera sesión inicial, el profesor introduce el tema, el material de lectura recomendado y el guión para su estudio. A partir de aquí, el proceso que se sigue es la elección del tema, el reparto de lecturas, trabajo personal del estudiante, y puesta en común para, finalmente, integrar las conclusiones de los debates en un trabajo escrito. A partir del curso académico 2005/2006 tuvimos la oportunidad de participar en la implementación de este método de enseñanza en la anterior Licenciatura en Derecho de la Universidad Miguel Hernández de Elche, donde nuestro maestro, el Profesor MIRÓ LLINARES, se planteó el objetivo de acercar a un conocimiento más profundo de la disciplina a aquellos estudiantes que mostraban una mayor motivación durante la docencia de las asignaturas "Derecho penal. Parte general" y "Derecho penal. Parte especial". Las temáticas abordadas por el mismo a lo largo de sus sucesivas ediciones fue diversa. El desarrollo de la primera edición (sobre los principios limitadores del Derecho penal) se estructuró de la siguiente forma: una vez establecido un calendario semanal de exposiciones, los estudiantes participantes preparaban la exposición de un tema con la ayuda de una selección de varias lecturas que les eran suministradasal inicio de las sesiones.

Tras la exposición individual se iniciaba un debate por parte del resto de participantes (incluidos los profesores que allí nos encontrábamos) sobre el contenido de la ponencia, una vez instruidos con las lecturas recomendadas y la exposición del ponente. El objetivo fundamental de esta fase era facilitar el acceso de los estudiantes por sus propios medios a un conocimiento más profundo del tema debatido, de tal forma que pudieran experimentar por sí mismos las ventajas e inconvenientes que se encuentra el operador jurídico a la hora de interpretar y aplicar el Derecho penal. Después de esta fase "interna", el seminario contemplaba una segunda fase en la que, una vez lograda la aproximación al conocimiento profundo de cada cuestión analizada, fueron 
invitados diversos Catedráticos y Profesores Titulares, que hubiesen tratado a lo largo de su trayectoria investigadora alguna de dichas cuestiones, con el objeto de que impartieran una conferencia a raíz de la cual se suscitaba un debate más enriquecedor de lo acostumbrado. Dada la gran aceptación que encontró en su primera edición, se celebró al curso siguiente la segunda, que seguía contando entre sus objetivos con el de afrontar la comprensión de problemas más o menos complejos del Derecho penal con métodos y procedimientos nuevos y diferentes a los tradicionalmente utilizados en la docencia de la, entonces, Licenciatura en Derecho. No obstante, a partir de aquel momento, la dinámica de trabajo se estructuró de forma diferente, convirtiendo en más dinámica y práctica la participación en el seminario. Concretamente, la metodología fue la siguiente: después de una sesión de presentación del seminario y una primera ponencia a cargo del Profesor MIRÓ LLINARES en la que se plantearon los presupuestos teóricos básicos del tema a analizar (en aquel caso, la autoría y la participación en el delito), y se entregaron los materiales fotocopiados con los que se debía trabajar, las siguientes sesiones trataban de resolver casos práctico-teóricos. Así, en los materiales de que disponían todos los miembros del seminario, había un calendario de sesiones y un conjunto de casos de este tipo. Cada semana, y después de que los miembros del seminario preparasen el caso con los materiales adjuntos a cada uno de ellos, se discutía el caso en común y se trataba de descubrir los principales puntos de polémica a plantear la semana siguiente. Una vez que el tema fue expuesto y discutido, asistía a la sesión siguiente el Doctor en Derecho penal especialista en el tema que presentaba el caso y exponía su solución al mismo, tras lo cual continuaba la discusión y se buscaba el acuerdo y la solución definitiva a los problemas planteados.

Con esa metodología modificada, pues, y pese al carácter, aparentemente, más técnico del tema que se había elegido, el seminario resultó más práctico, más participado y más provechoso que nunca, y marcó una línea para las subsiguientes ediciones, lo cual redundó en que la docencia cotidiana de Parte general y de Parte especial se viese impagablemente complementada con este método para la aplicación práctica de las nociones asimiladas por los estudiantes durante el período lectivo de la titulación oficial.

Sesiones de solución de problemas: o también llamada metodología de "Problem Based Learning" (Aprendizaje Basado en Problemas, ABP), sobre aplicación a la docencia del Derecho penal ya ha escrito ABEL SOUTO (2013: 90-106), y que tiene como característica primoridal que el estudiante debe resolver el problema que se le plantea partiendo exclusivamente de la información que sea esencial para el desarrollo de la práctica. Por ello, las sesiones de solución de problemas son consideradas por algunos autores como un tipo específico de seminario (GARCÍA BARBERO, ALFONSO, CANCILLO y CASTEJÓN, 1995). Los grupos deben ser de entre 5-10 estudiantes que seguirán un procedimiento establecido, como, por ejemplo, lectura del problema y análisis, encontrar definiciones o términos y conocimientos necesarios para su entendimiento y resolución, reparto del trabajo individualmente, búsqueda de documentación y estudio de los resultados conforme a las tareas establecidas.

Sesiones de demostración práctica: sirven para mostrar al estudiante cómo debe realizar una actividad práctica, fomentando, a su vez, el aprendizaje activo y proporcionándole información sobre sus logros. Para su utilización en la enseñanza práctica con- 
viene señalar que es más aconsejable su realización en grupos pequeños. En este sentido, si en la titulación de Derecho se reconociese un mayor índice de experimentalidad, se podrían realizar dichas sesiones con grupos de ese tamaño reducido y, por tanto, se facilitaría el seguimiento individualizado de los estudiantes en el proceso de identificación de los contenidos teóricos que se pretenden, así como la retroalimentación y valoración de resultados en la consecución de objetivos propuestos. Aquí podría incardinarse la técnica de simulación de juicios, como una de las propias de la metodología docente denominada "aprendizaje cooperativo" entre profesores y estudiantes (ALONSO RIMO, 2010; BLANCO CORDERO/SÁNCHEZ-MORALEDA VILCHES/SANDOVAL CORONADO, 2011).

Precisamente, para finalizar con las técnicas o métodos de enseñanza práctica, nos referiremos a las investigaciones propuestas por estudiantes o por profesores. En ellas se trata de identificar un problema objeto de estudio, formularlo con precisión y desarrollar los procedimientos adecuados para la obtención de resultados, interpretación y elaboración de conclusiones. Este aspecto de la investigación posiblemente es el que más hemos olvidado los docentes para nuestros estudiantes de Grado, y pensamos que sería muy interesante ya que, como muy bien ha afirmado el Profesor GONZÁLEZ RUS (2003: 6), cada vez más "el estudiante tiene un escaso contacto con los libros y con la doctrina y la jurisprudencia, limitando su conocimiento de éstas y de la asignatura a la visión que le proporciona su profesor". La realización de investigaciones es un buen instrumento, además, para la consecución de otro tipo de objetivos también importantes: el incentivar a nuestros estudiantes a utilizar las diferentes herramientas y técnicas que los capaciten en la búsqueda y solución de planteamientos teóricoprácticos poder realizar por sí mismos la interpretación y aplicación de los principios y normas penales, tales como las bases de datos, etc.

\subsubsection{Atención tutorial}

El profesor universitario, además de la carga docen te teórica y práctica de las asignaturas que imparte, debe tener programado un horario de tutorías semanales en las que estará disponible para la atención a sus estudiantes. En principio, las tutorías son un método y recurso para individualizar la enseñanza y ajustarla a las características personales de cada estudiante. En este sentido, la atención tutorial podría considerarse como la expresión máxima de la actividad docente, tanto en lo que ésta tiene de relación interpersonal como en la consecución de una enseñanza personalizada. Las tutorías permiten al profesor fomentary desarrollar las capacidades e intereses específicos de los alumnos ofreciendo retroalimentación sobre el trabajo que realizan. Por todo ello la tutoría se ha convertido en un espacio de tiempo en que el estudiante encuentra al profesor para cualquier asunto relacionado con la materia, aclaración de dudas, orientación de lecturas y trabajos individuales de carácter opcional, y finalmente, para la revisión de exámenes.

Las tutorías, como toda situación de enseñanza-aprendizaje, se han de planificar, realizar y evaluar de una manera sistemática siendo coherentes con los objetivos, contenidos y otras actividades del currículum del estudiante. También, si se orientan para resolver dudas de las exposiciones de las prácticas por ejemplo (programándolas de for- 
ma monográfica para cada una de las prácticas realizadas), las tutorías serían probablemente más demandadas. De este modo, se daría la opción de que el grupo de estudiantes asista a las diferentes tutorías específicas para cada práctica y resuelvan las dudas que les han surgido tras su realización. Por último, cabe destacar que en la actualidad, la práctica totalidad de las universidades españolas (y Centros de estudios como "Crímina" de la UMH, para el estudio prevención de la delincuencia, cuyo funcionamiento ha explicado MIRÓ LLINARES, 2013: 233-235) cuentan con un "Campus Virtual" que permite el contacto directo entre profesores y estudiantes para el intercambio de información, material docente, y resolución de las dudas que plantean los estudiantes vía correo electrónico (sobre el funcionamiento del Campus Virtual de la Universidad de Murcia, RODRÍGUEZ FERRÁNDEZ, 2013: 257-271). Éstos, además, suelen contar en sus universidades con aulas de informática de libre disposición, y de ordenadores personales localizados en lugares de acceso común para este fin.

\subsubsection{Recursos de aprendizaje}

Los recursos de aprendizaje o didácticos son los instrumentos que utiliza el docente para conseguir los objetivos previstos orientando y facilitando el aprendizaje de los estudiantes. De entre los diferentes recursos posibles a utilizar con el objetivo de favorecer la motivación y la eficacia del aprendizaje de los estudiantes, en concreto, y aplicado al ámbito del Derecho penal, es interesante destacar dos: la bibliografía y los recursos audiovisuales. Seleccionamos estos dos recursos porque el primero de ellos es, a nuestro juicio, el más comúnmente utilizado en Derecho, y porque el segundo todavía no es suficientemente utilizado por los profesores de nuestra disciplina (aunque va teniendo progresivamente una mayor presencia), y eso en un momento como el actual en el que el uso de las nuevas tecnologías ya no es una cuestión de utilidad sino de necesidad.

La bibliografía: en la que tienen una importancia creciente los manuales electrónicos (MORILLAS FERNÁNDEZ, 2013: 235-256), ha de ser exhaustiva y diversa, ya que los estudiantes deben poder acceder al conjunto de conocimientos relativos a las materias de estudio, por lo que es importante que esté reflejada en el programa de la asignatura y diferenciada por temas. Así los estudiantes, desde el inicio del curso académico ya pueden consultar con el profesor los aspectos más relevantes de la bibliografía de ca da tema. Es importante destacar que al decir "exhaustiva" queremos también decir "coherente", ya que no parece eficaz, de cara al estudiante, someterle a estímulos bibliográficos excesivamente inconexos, ya que entonces la bibliografía no orienta, sino que desorienta. Tiene que ser diversa porque es interesante que los estudiantes contrasten y comparen los planteamientos existentes respecto a diversos temas de autores que partan de principios de valor distintos.

Por otro lado, los medios audiovisuales constituyen unos materiales adecuados para acercar a los estudiantes a la comprensión de la realidad (sea a través de un caso real o, incluso, de un caso basado en la ciencia ficción ${ }^{9}$ ), de una forma cercana a sus intere-

\footnotetext{
${ }^{9}$ Como por ejemplo hizo MIRÓ LLINARES (2008: 27-41) con el caso sobre el que discurre la trama del filme "Minority Report", que utilizó para explicar el fundamento y contenido del principio de culpabilidad. También hay otros ejemplos como el de RÍOS CORBACHO (2012: 1-17) con "Blade Runner", el
} 
ses. Sin embargo, el mero hecho de usar medios audiovisuales no implica un proceso de innovación si previamente no se ha reflexionado sobre los propios medios y sobre cuál es el objeto de la innovación que se pretende. El uso de los medios en el proceso de enseñanza-aprendizaje, debe entenderse como una experiencia planificada y continua a lo largo de todo el curso. Lo mismo ocurre con la utilización de las nuevas tecnologías en nuestro trabajo docente. Herramientas como "Internet" y el aprendizaje de la correcta utilización de las bases de datos como "Westlaw", "El Derecho", V-Lex" o "Tirant lo Blanch" son poderosos instrumentos que los estudiantes han de dominar y que les permitirá adaptarse a los inevitables cambios que su cercana vida laboral les depara.

\subsubsection{Sistemas de evaluación}

Habitualmente se entiende por evaluación la valoración del aprendizaje de los alumnos, realizada tradicionalmente por el profesor como último responsable del proceso. Sin embargo, como hemos visto a lo largo del presente trabajo, la evaluación es un proceso en el que la recogida de información está en función de los objetivos, y sirve para tomar decisiones sobre todos los componentes implicados en el proceso de enseñanza-aprendizaje. De este modo, la evaluación, centrada habitualmente en calificar los conocimientos adquiridos por los estudiantes, únicamente refleja una parte del trabajo realizado a lo largo del curso académico. Es por ello que resulta del todo necesaria la inclusión de la evaluación continua, ofreciendo de este modo retroalimentación tanto al docente como al discente para plantear perspectivas y estrategias de mejora.

Como principio general, la evaluación debe estar basada en los aprendizajes obtenidos en función de los objetivos propuestos y, sobre todo, de las competencias planteadas en la ficha de materia y en la guía docente de la asignatura (específicamente sobre la evaluación de competencias en Derecho penal, CARRASCO ANDRINO, 2013: 173-182; DOMÍNGUEZ IZQUIERDO, 2013: 183-209; AGUILAR CÁRCELES, 2013: ). En este sentido, nos parece muy acertada la afirmación de GONZÁLEZ RUS (2003: 16), según el cual "el objetivo fundamental de los procedimientos de evaluación debe ser, más que el de acreditar la cantidad de información que el alumno es capaz de retenery reproducir, la comprobación del grado de formación alcanzado por el estudiante y su habilidad para analizar información, interpretarla y valorarla. En este sentido, la posibilidad de utilizar materiales de estudio (manuales, monografías, comentarios, apuntes, etc.), jurisprudencia y legislación vigente en las pruebas que se realicen debe considerarse el procedimiento ordinario".

Para finalizar vamos a describir brevemente los principales tipos de pruebas de evaluación empleadas, diferenciando entre la evaluación subjetiva frente a la objetiva, en ocasiones definidas como cualitativa frente a cuantitativa.

del mismo autor con películas como "La naranja mecánica", "La vida de Gale" o "Cadena Perpetua" (RÍOS CORBACHO, 2012: 6-23) para el estudio de las consecuencias jurídicas del delito, o el de HOLGADO SÁEZ (2013: 99-116) sugiriendo hasta siete películas distintas para la docencia del Derecho penal y del Derecho internacional. Combinando no sólo películas, sino también series de televisión, documentales y otros recursos audiovisuales, ABEL SOUTO (2012: 94-97). 


\section{A) Pruebas subjetivas}

Entre éstas se incluyen las pruebas de respuesta abierta a las que el estudiante responde de forma oral o escrita. Entre ellas, bien se pueden hacer preguntas más largas que abarquen casi un tema; bien que requieran el conocimiento del estudiante y también la capacidad de relacionar entre los distintos conceptos y elementos del sistema; o bien las pruebas PRAC (“Preguntas de Respuesta Abierta Corta”) limitadas a preguntas o definiciones cuya característica es la brevedad de la respuesta. Éstas últimas presentan como ventajas el requerir por parte del profesor poco tiempo para su preparación y el permitir evaluar la capacidad del estudiante para organizar y estructurar la información y resolver un problema. Sin embargo, presentan ciertos inconvenientes relacionados con la validez del contenido (muestreo insuficiente de los contenidos de aprendizaje), la confusión entre capacidad expresiva del alumno y dominio de los contenidos, o la fiabilidad interjueces si no están bien definidos los criterios de valoración de contenidos.

Para evitar en lo posible estos problemas, una vez que el profesor está convencido de que sus preguntas han sido formuladas correctamente y reflejan los contenidos y objetivos de aprendizaje, debe asegurarse de que él mismo o cualquier otra persona que corrija las repuestas lo hará con la mayor objetividad posible.

Queremos destacar en este apartado que, en el caso específico de la formación de egresados en Derecho en general, y en la formación en Derecho penal en particular, es necesario que se realicen evaluaciones orales y no sólo de forma escrita. Esto es así, pues, como ya hemos comentado, y siendo, además, afín a las directrices propuestas en el proceso de Convergencia Europea de nuestra titulación para la formación de un jurista, y por tanto, a tener en cuenta en su evaluación, hemos de valorar su capacidad de exposición oral y en público, así como su capacidad de debate de los argumentos jurídicos.

\section{B) Pruebas objetivas}

Se consideran pruebas de respuesta limitada, pues se imponen restricciones a la forma y contenido de las respuestas. Estas pruebas se clasifican en:

Pruebas de selección: el estudiante debe escoger entre las respuestas que se le ofrecen, aquélla que sea más adecuada a la pregunta. Incluyen preguntas de doble alternativa en las que sólo ofrecen dos posibilidades (verdadero-falso, sí-no, correctoincorrecto, etc.), o preguntas de elección múltiple en las que se ofrecen varias alternativas de las que sólo una es la más adecuada (escoger la única respuesta posible, elección de la opción incorrecta, o escoger la más apropiada). Las pruebas de elección múltiple permiten medir objetivos que requieren de la capacidad para inferir conclusiones, discriminar relaciones, e interpretar. Las ventajas de las pruebas de elección múltiple son la calificación objetiva, el control del azar, medir aprendizajes complejos y detectar áreas mal aprendidas a través del análisis de errores. Sin embargo, su mayor inconveniente es la dificultad de elaboración y es difícil controlar el efecto del azar. Esto último se puede lograr descontando al número de aciertos, el cociente de dividir el número 
de errores por el número de alternativas de cada pregunta menos 1 , expresado en la siguiente fórmula: Puntuación = Aciertos - (Errores/núm. alternativas-1). Es decir que, en un examen test, lo ideal puede ser una pregunta con cinco alternativas en las que cada cuatro mal elimina una bien.

Evaluación de mapas conceptuales: en este tipo de pruebas de evaluación, el estudiante debe desarrollar a partir de un concepto clave, los contenidos de un tema o de una unidad temática, construyendo un mapa en el que se reflejan todos los conceptos y relaciones que sean capaces de asociar. Entre sus ventajas está el ser adecuados para evaluar objetivos de diferenciación e integración conceptual. Como inconveniente, su corrección es costosa, pues requiere puntuar atendiendo a las relaciones y niveles jerárquicos válidos, los nexos que supongan integración de conceptos y, en su caso, ejemplos, estando así sujetas a cierto grado de subjetividad.

En nuestra opinión es muy interesante el combinar diferentes modelos de evaluación en la misma prueba en la valoración de los contenidos teóricos. De esta forma, se puede dar la oportunidad al estudiante de poder demostrar su conocimiento no sometiendo la valoración del mismo a un tipo de pruebas en la que quizá no sea muy diestro. Así mismo, consideramos que es un buen método de evaluación en nuestras asignaturas la combinación de preguntas cortas con el mapa conceptual, siempre, claro está, en función de los objetivos propuestos en dicha evaluación.

Para la evaluación de los contenidos prácticos de una asignatura se puede utilizar alguna de las pruebas objetivas o subjetivas mencionadas; en este último caso, por ejemplo, un modo de hacerlo es mediante la elaboración de informes de prácticas y proyectos, dictámenes o pequeños de trabajos de investigación. También la evaluación de prácticas puede realizarse a través de la observación de los estudiantes durante su trabajo práctico o exposición del mismo, y de las opiniones que éstos tienen sobre la organización y realización de las prácticas.

\section{Recapitulación y reflexión final}

A lo largo de las páginas precedentes hemos tratado de exponer cuál es nuestro propio modelo de enseñanza-aprendizaje del Derecho Penal exponiendo en primer lugar los fundamentos que lo inspiran para, a continuación, explicar pormenorizadamente las técnicas de metodología docente, teórica y práctica, que aplicamos, así como los principales recursos de los que nos valemos. Y ello refiriéndonos puntualmente, además, a diferentes experiencias innovadoras en las que hemos participado en este ámbito.

Con ello queremos destacar finalmente que, ante todo, lo que debe quedar claro es que hemos de tener muy presente la importancia de ser partícipes de la constante innovación en la impartición de la docencia universitaria (SUÁREZ LÓPEZ, 2014) ante el escenario en el que nos ha situado el Espacio Europeo de Educación Superior (RíOS CORBACHO, 2012; VÁZQUEZ-PORTOMEÑE SEIJAS/PÉREZ RIVAS, 2012), un escenario quizá impensable para muchos hace unos años, pero para el que otros ya nos veníamos preparando y ante el que nos presentamos sin prejuicios y con las máximas 
inquietudes y ganas de seguir mejorando, en la vertiente docente de nuestra labor diaria en los centros universitarios.

\section{Bibliografía}

ABEL SOUTO, M. (2010). "Derecho penal, norma de valoración, bien jurídico y enseñanza de valores en el EEES". REJIE: Revista Jurídica de Investigación e Innovación Educativa, 2, pp. 93-100.

ABEL SOUTO, M. (2012). "Desde la literatura, ópera y televisión hasta las ciencias jurídicas y el Derecho penal mediante la nueva técnica pedagógica del aprendizaje basado en problemas". REJIE: Revista Jurídica de Investigación e Innovación Educativa, 5, pp. 87-104.

ABEL SOUTO, M. (2013). “Metodologías docentes activas en Derecho Penal y puesta a disposición de recursos de aprendizaje que faciliten el trabajo autónomo". D.L. MoriIlas Fernández (Dir.) y S. Rodríguez Ferrández (Coord.). Innovación docente y Derecho Penal. Murcia: EditUM, pp. 90-106.

AGUILAR CÁRCELES, M.Ma . (2013). "Evaluación por competencias: la matriz de valoración aplicada al Derecho Penal”. D.L. Morillas Fernández (Dir.) y S. Rodríguez Ferrández (Coord.). Innovación docente y Derecho Penal. Murcia: EditUM, pp. 210-227.

ALONSO RIMO, A. (2010). "Aprendizaje cooperativo en Derecho Penal: algunas estrategias". D.L. C. Gómez Lucas y S. Grau Company (Coords.). Evaluación de los aprendizajes en el Espacio Europeo de Educación Superior. Alicante: Marfil, pp. 205-219.

AUSUBEL, D.P. (1963). The Psychology of Meaningful Verbal Learning. New York: Grune \& Stratto.

BERNAL DEL CASTILLO, J. (2014). "El proyecto de Intercambio de movilidad docente Erasmus en el ámbito de las Ciencias Penales (Experiencias desde la Universidad de Oviedo)". REJIE: Revista Jurídica de Investigación e Innovación Educativa, 10, pp. 127140.

BLANCO CORDERO, I./SÁNCHEZ-MORALEDA VILCHES, N./SANDOVAL CORONADO, J.C. (2011). "El trabajo colaborativo en la asignatura Derecho Penal-Parte General (Licenciatura en Derecho-Facultad de Derecho)". Ma.T. Tortosa Ybáñez, J.D. Álvarez Teruel y N. Pellín Buades (Coords.). IX Jornades de xarxes d'investigació en docència universitària: Disseny de bones pràctiques docents en el context actual. Alicante: Universidad de Alicante, pp. 305-315.

BOIX REIG, J./ORTS BERENGUER E. (1997). "Divagaciones sobre las relaciones entre teoría y práctica en el mundo del Derecho, en particular en el mundo del Derecho penal". E. Orts Berenguer (Ed.). Estudios jurídicos: Libro Homenaje al Profesor José Ramón Casabó Ruiz. Volumen I. Valencia: Servicio de Publicaciones de la Universidad de Valencia, pp. 201-225.

BRUNER, J.S. (1961). The process of education. A landmark in educational theory. 
Cambridge: Harvard University Press.

CARIO, R./DE LA CUESTA ARZAMENDI, J.L. (1990). "El programa Erasmus 'Derecho Penal y Ciencias Criminales: nuevas políticas penales en Europa'. Una experiencia de integración potencial de la enseñanza de la Criminología en Europa". Eguzkilore, 3 (extraordinario), pp. 185-196.

CARRASCO ANDRINO, Mạ. del M. (2013). "Evaluación de competencias: un reto en el EEES". D.L. Morillas Fernández (Dir.) y S. Rodríguez Ferrández (Coord.). Innovación docente y Derecho Penal. Murcia: EditUM, pp. 173-182.

DE LA CRUZ TOMÉ, Mạ.Á. (1981). Interacción en el aula universitaria. Madrid: INCIE.

DE PABLOS PONS, J. (1988). "Estrategias de enseñanza y medios instructivos". J. De Pablos Pons (Ed.). El trabajo en el aula. Sevilla: Alfar.

DOMÍNGUEZ IZQUIERDO, E.Mạ. (2013). "Evaluación de competencias: un reto en el EEES". D.L. Morillas Fernández (Dir.) y S. Rodríguez Ferrández (Coord.). Innovación docente y Derecho Penal. Murcia: EditUM, pp. 183-209.

GONZÁLEZ GARCÍA, F.Ma./IBÁÑEZ, F.C./CASALÍ, J. (2000). Una aportación a la mejora de la calidad de la docencia universitaria: los mapas conceptuales. Pamplona: Universidad Pública de Navarra.

GONZÁLEZ RUS, J.J. (2003). "Reflexiones sobre el futuro de la enseñanza del Derecho y sobre la enseñanza del Derecho en el futuro". Revista Electrónica de Ciencia Penal y Criminología, 5, pp. 1-21.

HOLGADO SÁEZ, C. (2013). "Derecho y cine del genocidio: 7 títulos contemporáneos (2001-2011) para la docencia presencial del Derecho Penal e Internacional Público". REJIE: Revista Jurídica de Investigación e Innovación Educativa, 8, pp. 100-116.

ÍNNIGO CORROZA, E./SÁNCHEZ-OSTIZ GUTIÉRREZ, P. (2014). "Sobre el uso del método inductivo en la enseñanza del Derecho (Penal)". REJIE: Revista Jurídica de Investigación e Innovación Educativa, 9, pp. 9-22.

MIRÓ LLINARES, F. (2008). “¿Sueñan los androides con la libertad de los hombres? Un comentario jurídico penal a Minority Report". A. Ortega Giménez y P. Crema des García (Coords.). Cine y Derecho en 13 películas. San Vicente: Editorial Club Universitario, pp. 27-41.

MIRÓ LLINARES, F. (2013). "E-learning aplicado a las Ciencias Penales". D.L. Morillas Fernández (Dir.) y S. Rodríguez Ferrández (Coord.). Innovación docente y Derecho Penal. Murcia: EditUM, pp. 228-234.

MIRÓ LLINARES, F. (Dir.)/RODRÍGUEZ FERRÁNDEZ, S. (Coord.) (2010). ¿Casos difíciles o irresolubles? Problemas esenciales de la Teoría del Delito desde el análisis de paradigmáticos casos jurisprudenciales. Madrid: Dykinson.

MORILLAS CUEVA, L. (2013). “La adaptación del sistema universitario español al EEES. 
El Grado en Derecho y el Derecho penal". D.L. Morillas Fernández (Dir.) y S. Rodríguez Ferrández (Coord.). Innovación docente y Derecho Penal. Murcia: EditUM, pp. 11-44.

MORILLAS FERNÁNDEZ, D.L. (2013). "El libro electrónico como herramienta de enseñanza del Derecho Penal”. D.L. Morillas Fernández (Dir.) y S. Rodríguez Ferrández (Coord.). Innovación docente y Derecho Penal. Murcia: EditUM, pp. 235-256.

NIETO MARTÍN, A. (2008). "Guía para la realización de casos prácticos en la asignatura de Derecho Penal”. Multiárea: Revista de Didáctica, 3, pp. 333-344.

PÉREZ LLEDÓ, J.A. (1992). "La enseñanza del Derecho en Estados Unidos”. Doxa. Cuadernos de Filosofía del Derecho, 12, pp. 41-94.

PERIS RIERA, J. et al. (2013). Manual docente: Casos Prácticos de Derecho Penal Parte General. Murcia: EditUM.

PUENTE FERRERAS, A. (2003). Cognición y aprendizaje. Fundamentos psicológicos. Madrid: Pirámide.

RÍOS CORBACHO, J.M. (2012). “Blade runner: Derechos humanos, ingeniería genética y derecho penal a través del cine". ASRI: Arte y Sociedad. Revista de investigación, 2 , pp. 1-17.

RÍOS CORBACHO, J.M. (2012). "Las consecuencias jurídicas del delito y el cine: un acercamiento a la resocialización en el nuevo siglo". Ciencia ergo sum, 19-1, pp. 6-23.

RÍOS CORBACHO, J.M. (2012). "Sobre la metodología y herramientas en la enseñanza del moderno Derecho Penal". REJIE: Revista Jurídica de Investigación e Innovación Educativa, 6, pp. 55-80.

RODRÍGUEZ FERRÁNDEZ, S. (2013). "Algunas utilidades del 'Aula Virtual' para la docencia en el marco del EEES". D.L. Morillas Fernández (Dir.) y S. Rodríguez Ferrández (Coord.). Innovación docente y Derecho Penal. Murcia: EditUM, pp. 257-271.

SÁINZ CANTERO, J.A. (1983-1984). "La enseñanza del Derecho Penal y la Criminología a través del análisis de causas penales". Estudios Penales y Criminológicos, 8, pp. 279298.

SUÁREZ LÓPEZ, J.Mạ. (2014). “La importancia del empleo de las metodologías innovadoras en la enseñanza del Derecho Penal”. Anales de Derecho, 32, pp. 1-32.

TORío LÓPEZ, Á. (2004). “Misión actual de la Facultad de Derecho”. J. López Barja de Quiroga y J.M. Zugaldía Espinar (Coords.). Dogmática y Ley Penal. Libro homenaje a Enrique Bacigalupo. Tomo I. Madrid/Barcelona: Instituto Universitario de Investigación Ortega y Gasset/Marcial Pons, pp. 917-927.

VÁZQUEZ-PORTOMEÑE SEIJAS, F./PÉREZ RIVAS, N. (2012). "Técnicas de enseñanzaaprendizaje del Derecho penal en el marco del EEES". Anuario da Facultade de Dereito da Universidade da Coruña, 16, pp. 647-659. 
